\title{
Atuação fonoaudiológica em home care
}

\author{
Phonoaudiological performance in home care \\ Desempeño fonoaudiológico en el cuidado doméstico
}

Recebido: 30/12/2020 | Revisado: 31/12/2020 | Aceito: 07/01/2021 | Publicado: 09/01/2021

\author{
Keyla Maria da Rocha Silva \\ ORCID: https://orcid.org/0000-0002-6685-4944 \\ Faculdade de Ensino Superior do Piauí, Brasil \\ E-mail: Keyla.rocha.18@gmail.com \\ Danilo Sampaio \\ ORCID: https://orcid.org/0000-0002-0216-3669 \\ Faculdade de Ensino Superior do Piauí, Brasil \\ E-mail: Danilosampaio16@hotmail.com
}

\begin{abstract}
Resumo
O fonoaudiólogo em Home care atua gerenciando as necessidades dos pacientes, como por exemplo, em disfagias, realizando avaliações contínuas, além de especificar os métodos mais adequados de tratamento. O objetivo geral da presente pesquisa consiste em analisar a importância da atuação do fonoaudiólogo no ambiente domiciliar. Dentre os objetivos específicos ressaltam-se: identificar qual o papel do fonoaudiólogo no home care; verificar quais os benefícios para o paciente em realizar o tratamento em casa; demonstrar quais as principais demandas da atuação do fonoaudiólogo em home care. A problemática da pesquisa reside no seguinte questionamento: qual a importância da atuação fonoaudiológica na residência dos pacientes? No que se refere a metodologia a pesquisa pode ser caracterizada como uma revisão bibliográfica do tipo integrativa, de análise qualitativa, tendo como intuito a sistematização de resultados de fontes teóricas variadas sobre a presente temática, em especial artigos disponibilizados em bases de dados direcionadas a pesquisa científica, como Scielo, Capes, Scholar. Evidenciou-se por meio dos estudos encontrados que o fonoaudiólogo é de suma importância para um atendimento personalizado, pois proporciona a diminuição dos riscos de internações, evitando, dessa forma, exposições ao ambiente hospitalar, com a privacidade do leito familiar, facilitando o tratamento, pois o apoio emocional é de fundamental importância para a recuperação.
\end{abstract}

Palavras-chave: Fonoaudiólogo; Home care; Tratamento.

\begin{abstract}
The speech therapist in Home care works by managing the needs of patients, such as, for example, in dysphagia, performing continuous evaluations, in addition to specifying the most appropriate methods of treatment. The general objective of this research is to analyze the importance of the performance of the speech therapist in the home environment. Among the specific objectives, the following stand out: to identify the role of the speech therapist in home care; verify the benefits to the patient in carrying out the treatment at home; demonstrate what are the main demands of the speech therapist in home care. The research problem lies in the following question: what is the importance of speech therapy in patients' homes? Regarding the methodology, the research can be characterized as a bibliographic review of the integrative type, of qualitative analysis, aiming at the systematization of results from varied theoretical sources on the present theme, especially articles available in databases directed to research scientific, such as Scielo, Capes, Scholar. It was evidenced through the studies found that the speech therapist is of paramount importance for personalized care, as it provides a reduction in the risks of hospitalizations, thus avoiding exposures to the hospital environment, with the privacy of the family bed, facilitating treatment, because emotional support is of fundamental importance for recovery.
\end{abstract}

Keywords: Speech therapist; Home care; Treatment.

\section{Resumen}

El logopeda en Atención domiciliaria trabaja gestionando las necesidades de los pacientes, como por ejemplo en la disfagia, realizando evaluaciones continuas, además de concretar los métodos de tratamiento más adecuados. El objetivo general de esta investigación es analizar la importancia de la actuación del logopeda en el ámbito del hogar. Entre los objetivos específicos destacan: identificar el papel del logopeda en la atención domiciliaria; verificar los beneficios para el paciente al realizar el tratamiento en casa; demostrar cuáles son las principales demandas del logopeda en la atención domiciliaria. El problema de la investigación radica en la siguiente pregunta: ¿cuál es la importancia de la logopedia en los hogares de los pacientes? En cuanto a la metodología, la investigación se puede caracterizar como una revisión bibliográfica de tipo integrador, de análisis cualitativo, con el objetivo de sistematizar resultados de variadas fuentes teóricas sobre la presente temática, especialmente artículos disponibles en bases de datos dirigidas a la investigación. 
científicos, como Scielo, Capes, Scholar. Se evidenció a través de los estudios hallados que el logopeda es de suma importancia para la atención personalizada, ya que brinda una reducción en los riesgos de hospitalizaciones, evitando así exposiciones al ambiente hospitalario, con la privacidad de la cama familiar, facilitando el tratamiento, porque el apoyo emocional es de fundamental importancia para la recuperación.

Palabras clave: Logopeda; Cuidados en el hogar; Tratamiento.

\section{Introdução}

Na atenção domiciliar, existem dois setores de serviços: o público e o privado, o atendimento domiciliar na rede pública denominado Serviço de Atenção Domiciliar (SAD) surgiu com o objetivo de minimizar os agravos encontrados no Sistema Único de Saúde (SUS) e prestar atendimento para promoção, prevenção e humanização. No setor privado, a atenção domiciliar tem como objetivo promover, manter e / ou restaurar a saúde (Goulart, 2010).

O Decreto $n^{\circ} 963$ de 27 de maio de 2013 preceitua de forma expressa sobre os objetivos da atenção domiciliar, conforme pode ser observado em seu art. 3, o qual afirma que esse tipo de Atenção em domicílios tem como objetivo a reorganização do processo de trabalho das equipes que prestam cuidado domiciliar na atenção básica, ambulatorial, nos serviços de urgência e emergência e hospitalar, com vistas à redução da demanda por atendimento hospitalar e/ou redução do período de permanência de usuários internados, a humanização da atenção, a desinstitucionalização e a ampliação da autonomia dos usuários (Brasil, 2013).

O ambiente hospitalar é muito debilitante e pode dificultar o sucesso do tratamento. Portanto, o número de pessoas que procuram atendimento domiciliar está aumentando. O Ministério da Saúde do Brasil descreveu que a atenção domiciliar é uma nova forma de cuidar da saúde que substitui ou complementa os métodos existentes. Caracteriza-se por proporcionar um conjunto de açães de promoção da saúde, prevenção e tratamento de doenças e reabilitação no domicílio, e garante a continuidade do cuidado da rede médica por meio de uma abordagem integral (Padovani, 2013).

Os serviços de atendimento domiciliar começaram nos Estados Unidos da América (EUA) na década de 1950, com foco principalmente na redução de custos. Em 1965, o procedimento foi modificado para beneficiar os pacientes. Surgiu no Brasil e no Estado de São Paulo em 1967, tendo como objetivo reduzir o número de leitos do Hospital Servidor Público Estadual e oferecer tratamento extra-hospitalar para pessoas com doenças crônicas (Ribeiro, 2011).

Segundo o Ministério da Saúde do Brasil o SAD é composto por uma equipe multidisciplinar composta por médicos, enfermeiros, fisioterapeutas, nutricionistas, psicólogos, assistentes sociais, fonoaudiólogos, dentistas, farmacêuticos e terapeutas ocupacionais. De acordo com a Portaria n 963 de 27 de maio de 2013, o número mínimo de grupos multiprofissionais de apoio (EMAP) é de três profissionais de ensino superior selecionados nas ocupações acima (Brasil,2013).

Em 2008, foi criado o Núcleo de Apoio à Saúde da Família (NASF) com o objetivo de ampliar a abrangência das ações da atenção básica e suas soluções para apoiar a inserção da Estratégia Saúde da Família (ESF) na atenção à saúde da família. Devido ao aumento dos pacientes atendidos no ambiente familiar, as pessoas estão cada vez mais conscientes de que os idosos precisam se beneficiar deste serviço (Ribeiro, 2011).

No Brasil, a prevalência de idosos sob cuidados domiciliares é alta. Um dos fatores mais comuns são os transtornos, doenças crônicas e disfagia, que podem levar à pneumonia aspirativa e desnutrição. A inserção da fonoaudiologia na intervenção familiar ainda é recente e existem poucos estudos sobre o assunto (Cardoso, 2012).

O objetivo geral da presente pesquisa consiste em analisar a importância da atuação do fonoaudiólogo no ambiente domiciliar. Dentre os objetivos específicos ressaltam-se: identificar qual o papel do fonoaudiólogo no home care; verificar quais os benefícios para o paciente em realizar o tratamento em casa; demonstrar quais as principais demandas da atuação do fonoaudiólogo em home care; Demonstrar O perfil do usuário atendido pelo profissional de fonoaudiologia em home care 
A problemática da pesquisa reside no seguinte questionamento: qual a real importância da atuação dos profissionais de Fonoaudiologia na residência dos pacientes?

Tendo em vista a função da discussão do tema, nesse sentido, completando a fundamentação de forma completa e proporcionando recursos estratégicos, este trabalho distrai do problema de pesquisa subsequente: como se dá a atuação do fonoaudiólogo em home care?

Esta pesquisa mostra relevância em traçar um panorama da assistência domiciliar com enfoque nas possibilidades de atuação fonoaudiológica, sendo instrumento norteador de quem deseje trabalhar nesta área ou comparar com outros serviços. Constitui-se também como dado oficial (não empírico) para correlações futuras ou presentes e de outros municípios, uma vez constatada a escassez de material publicado neste tema. Tornando-se relevante para comunidade acadêmica, uma vez que enrique ainda mais a produção científica acerca desse tema. Por sua vez, estabelece uma base para referenciar todas as fundações e seu valor acumulado, ou seja, a base científica é agregada por meio do embasamento estudado.

\section{Referencial Teórico}

\subsection{Noções gerais sobre o home care}

A atenção domiciliar é definida por Moreira; Martins e Castro (2012), como um conjunto de serviços realizados no domicílio com os pacientes, esses serviços visam fornecer-lhes suporte terapêutico. Este suporte varia de cuidados pessoais (por exemplo, banho, alimentação, roupas e higiene pessoal) a cuidados médicos, seja cirurgia ou curativo, manipulação de tecnologia hospitalar (por exemplo: respiradores), nutrição enteral/parenteral, diálise e outros. Esses serviços são prestados por médicos e enfermeiras plantonistas 24 horas por dia, além de uma equipe de apoio.

O objetivo do atendimento domiciliar é promover a liberação de leitos hospitalares e ambulatoriais para que haja uma redução de custos, reintegrar o paciente à família e apoiar o núcleo, realizar um trabalho mais humano e holístico, estimular o paciente e sua família a participarem mais do tratamento e reduzir os hospitais. $\mathrm{O}$ atendimento domiciliar também é indicado para pacientes com dificuldades ou sem condições de mobilidade porque não podem se locomover por causa de alguma patologia ou qualquer outra condição e, portanto, não podem receber tratamento em uma clínica, consultório ou hospital (Zeppe, 2014).

Para a realização desse trabalho, o serviço de atenção domiciliar conta com uma equipe de saúde composta por diversos profissionais de diferentes profissões, que podem atender às necessidades dos pacientes que necessitam desse serviço. Essa equipe é caracterizada por uma equipe multidisciplinar que pode contar com diversos profissionais, como médicos (todas as habilitações), enfermeiros, técnicos de enfermagem, serviço social, nutrição, fisioterapia, fonoaudiologia, odontologia, terapia ocupacional, farmácia e psicologia (Zeppe, 2014).

Nos últimos dez anos, o programa de atendimento domiciliar (AD) foi ampliado internacionalmente e implantado no setor hospitalar por meio do processo de desospitalização, o que reduz muito os riscos de infecção hospitalar, economiza gastos com internação, racionaliza a demanda por leitos hospitalares, dá mais conforto ao paciente e sua família e torna o atendimento mais humano (Vasconcelos et. al. 2015).

O objetivo da inclusão da AD no SUS é garantir que os pacientes e seus familiares cumpram os princípios e diretrizes constitucionais de universalidade, integridade, justiça, participação da comunidade, descentralização, regionalização, hierarquia e atender às necessidades crescentes dos demais (Braga et. al. 2016).

A AD é composto por uma série de condições que dificultam a compreensão dos profissionais de saúde e, diante dessas situações, as pessoas têm proposto formas de especificar diversas medidas de cuidado no ambiente domiciliar. Uma vez que abrange outras definições e considera todos os objetivos ao mesmo tempo, é dividido em cuidados domiciliares mais abrangentes, visitas domiciliares e AD (Gallassi et. al. 2014). 
A AD sugere que os cuidados de enfermagem sejam realizados de forma personalizada e de acordo com a situação real do paciente para poder restabelecer a saúde. Ao receber cuidados dos profissionais, além de promover o salvamento das relações interpessoais, o paciente também tende a ter uma visão de melhora, prolongar sua vida com qualidade, compreender sua real condição clínica, suas visões de futuro e autonomia própria (Silva et. al. 2017).

$\mathrm{O}$ atendimento domiciliar pode variar de acordo com as necessidades dos indivíduos, sejam eles em período integral ou apenas prestando ajuda e suporte no cuidado de idosos vulneráveis, deficientes e adultos em curto, médio e longo prazo (Genet et. al. 2011).

Segundo Silva et. al. (2013, p. 14), o atendimento médico domiciliar pode representar um movimento em busca de um novo modelo de atendimento médico integral e justo, garantindo a qualidade de vida e estimulando a autonomia individual, configurando-o como estratégia descentralizada de atendimento médico.

As vantagens do Atendimento Domiciliar consiste em proporcionar ao paciente conforto, participação e interação com a família e demais pessoas do círculo social. Determinou-se que para os familiares envolvidos no cuidado ao paciente será mais confortável e menos estressante em casa (Borges et. al. 2016).

\subsection{O perfil do usuário atendido pelo profissional de fonoaudiologia em home care}

Do ponto de vista das equipes e ações de saúde da família (crianças), diante das necessidades fonoaudiológicas da população voltadas para o desenvolvimento da função do sistema sensório-motor oral, os distúrbios de linguagem oral infantil são os mais evidentes (Wachs et al. 2016).

Nos resultados sociodemográficos dos usuários do SAD, o desempenho individual dos homens $(59,6 \%)$ e dos idosos $(62,3 \%)$ foi destacado, corroborando outros resultados da literatura. Esses estudos mostram que, em termos de variáveis sociodemográficas e clínicas, a maioria são homens, idosos, brancos, casados e pessoas com ensino fundamental incompleto. Porém, outro estudo realizado em âmbito nacional constatou que a identidade dos usuários na atenção domiciliar está relacionada à mulher, doenças crônicas e disfunções (Alves \& Barbosa, 2010).

Portanto, os dados sugeridos podem estar relacionados às características epidemiológicas típicas de cada região. Além disso, a vantagem da população idosa pode ser explicada pela incapacidade causada por doenças que acometem essa faixa etária. Esses dados estão relacionados ao aumento da expectativa de vida e às doenças crônicas nessa faixa etária, o que torna ainda mais evidente a importância do atendimento domiciliar, principalmente porque muitas pessoas têm dificuldade de se locomover (Moreira et. al. 2014).

Em um estudo de revisão bibliográfica, Ferreira; Bansi e Paschoa (2014, p.32) descrevem os serviços de atendimento domiciliar relacionados às necessidades do usuário, procedimentos de atendimento e gerenciamento de serviços. Eles descobriram que devido ao envelhecimento da população, a demanda por cuidados para os idosos tende a aumentar.

\section{Metodologia da Pesquisa}

Trata-se de um estudo de revisão integrativa da literatura que, de acordo com Freire et al. (2014, p.11) tem sido utilizada como recurso metodológico, a qual faz uso de estratégia sistematizada para agrupar e produzir resultados de estudos sobre um determinado tema, com o objetivo de aprofundar e reforçar o conhecimento científico de determinadas áreas e subsidiar a tomada de decisões dos profissionais.

Para a construção de uma revisão integrativa é necessário seguir seis etapas distintas: a identificação do tema e seleção da hipótese ou questão de pesquisa; estabelecimento de critérios para inclusão e exclusão de estudos/amostragem ou busca na 
literatura; definição das informações a serem extraídas dos estudos selecionados/categorização dos estudos; avaliação dos estudos incluídos; interpretação dos resultados; e apresentação da revisão/síntese do conhecimento (Ercole, Melo \& Alcoforado, 2014).

O estudo foi realizado pela busca de dados nas bases capes, scholar e scielo com os seguintes descritores: Fonoaudiologia. Home care. Atuação fonoaudiológica. Os critérios de inclusão foram os artigos que apresentavam texto completo e em português os quais se encontravam no período de 2010 a 2020 e que estavam relacionados ao tema. E os critérios de exclusão foram textos que não apresentaram relação com a temática, revisões, livros e os artigos fora do recorte temporal.

Foi classificada a combinação dos artigos selecionados, analisando o título e o resumo de cada artigo. A posição de assunto destes artigos (para verificação) leva em consideração documentos em português e inglês, com publicações feitas nos últimos 10 anos. Depois de realizada a busca na base de dados encontraram-se 250 artigos relacionados aos descritores. Ao filtrar para artigos em português, resultaram em 190, destes, foram excluídos 134 ao fazer o filtro com texto completo. Posteriormente, foram selecionados aqueles que apresentaram relação com a temática, ficando assim 56 artigos, sendo excluídos 45 artigos por não se enquadrarem no recorte temporal, o que resultou em uma amostra final de 14 artigos, conforme a Figura 1:

Figura 1. Quantidade de artigos conforme a base de dados.

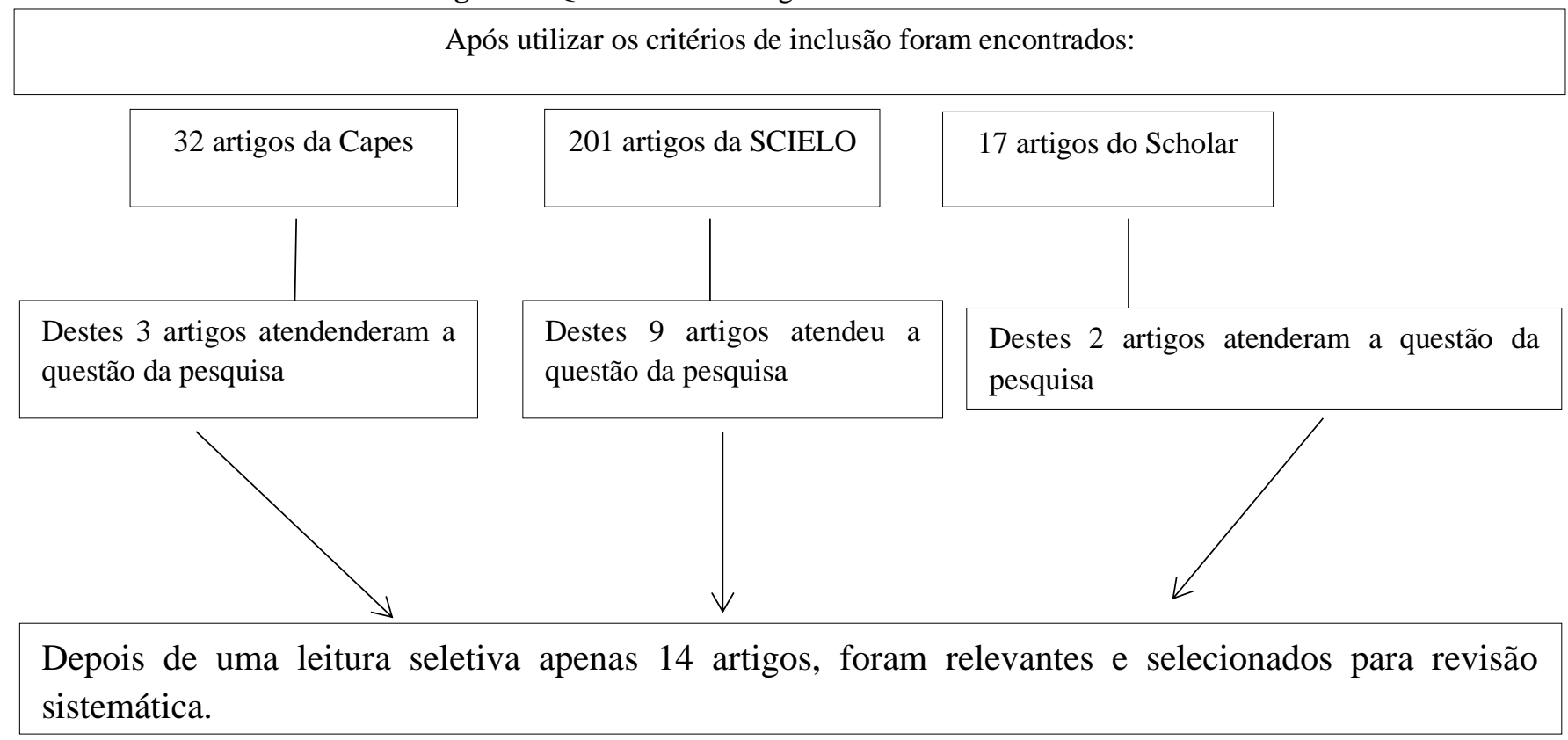

Fonte: Autores (2020).

\section{Resultados e Discussão}

Os estudos trabalhados foram classificados para demonstrar a contribuição dos mesmos em relação à atuação do fonoaudiólogo em home care, conforme está descrito no Quadro 1. 
Quadro 1. Classificações dos estudos segundo Título do artigo, Autores e Contribuição.

\begin{tabular}{|c|c|c|}
\hline Título do Artigo & Autor & Contribuição \\
\hline $\begin{array}{l}\text { Aged care services and home } \\
\text { health care and institutional } \\
\text { strategies }\end{array}$ & $\begin{array}{l}\text { Fereira, FPC; Bansi, LO.; } \\
\text { Paschoal, SMP. }\end{array}$ & $\begin{array}{l}\text { O incentivo à implantação de serviços que possam referenciar as } \\
\text { demandas das regiões é fundamental para atingir a população idosa } \\
\text { em seus diversos aspectos, através de uma equipe } \\
\text { multiprofissional. }\end{array}$ \\
\hline $\begin{array}{l}\text { Atenção domiciliar na } \\
\text { atenção primária à saúde: } \\
\text { uma síntese operacional. }\end{array}$ & Gallasi, C.V.; et al & $\begin{array}{l}\text { A avaliação e a reconstrução contínuas das estratégias de cuidado } \\
\text { por meio de fóruns e conferências catalisadores de trocas de } \\
\text { experiências e de oportunidades para a formação teórica e prática } \\
\text { dos profissionais envolvidos }\end{array}$ \\
\hline $\begin{array}{l}\text { Home care in Europe: a } \\
\text { systematic literature review }\end{array}$ & Genet, N. et al & $\begin{array}{l}\text { Mostra claramente a necessidade de mais publicações científicas } \\
\text { sobre atenção domiciliar, principalmente estudos comparando } \\
\text { países. Uma visão abrangente e mais completa sobre o estado dos } \\
\text { cuidados domiciliares na Europa requer a coleta de informações } \\
\text { usando uma estrutura e metodologia uniformes. }\end{array}$ \\
\hline $\begin{array}{l}\text { Por que é melhor em casa? A } \\
\text { percepção de usuários e } \\
\text { cuidadores da atenção } \\
\text { domiciliar. }\end{array}$ & Silva, KL. et al. & $\begin{array}{l}\text { Analisar as perspectivas dos usuários sobre os atendimentos de } \\
\text { suas necessidades pelo serviço de Atenção Domiciliar }\end{array}$ \\
\hline $\begin{array}{l}\text { Desigualdades na } \\
\text { mortalidade por doenças } \\
\text { crônicas entre idosos e sua } \\
\text { associação com indicadores } \\
\text { socioeconômicos no Brasil }\end{array}$ & $\begin{array}{l}\text { Alves, DB, } \\
\text { Barbosa, MTS }\end{array}$ & $\begin{array}{l}\text { Cuidados paliativos referem-se à melhoria da qualidade de vida } \\
\text { dos pacientes e familiares, auxiliando no enfrentamento dos } \\
\text { problemas relacionados à doença }\end{array}$ \\
\hline $\begin{array}{l}\text { Hemorrhagic complications } \\
\text { associated with dabigatran } \\
\text { use }\end{array}$ & Chen, BC. et al & $\begin{array}{l}\text { A morte deve ser considerada um processo natural que não será } \\
\text { acelerada e nem adiada }\end{array}$ \\
\hline $\begin{array}{l}\text { Presbifagia: estado da arte da } \\
\text { deglutição do idoso. }\end{array}$ & Cardoso, MCAF & $\begin{array}{l}\text { Para a equipe, pacientes e familiares, nem sempre é fácil aceitar } \\
\text { todas as possibilidades de cura da doença por meio do tratamento, } \\
\text { e o indivíduo se encontra em situação crítica de } \\
\text { Terminalidade }\end{array}$ \\
\hline $\begin{array}{l}\text { Redes organizacionais como } \\
\text { alternativa para gestão } \\
\text { hospitalar: o sindicato dos } \\
\text { hospitais beneficentes, } \\
\text { religiosos e filantrópicos do } \\
\text { Vale do Rio Pardo } \\
\text { (SINDHVARP). }\end{array}$ & Fontoura, VT ; Arend, SC & $\begin{array}{l}\text { Os fonoaudiólogos são membros ativos e importantes da equipe } \\
\text { multiespecialidade de cuidados paliativos. }\end{array}$ \\
\hline
\end{tabular}




\begin{tabular}{|c|c|c|}
\hline $\begin{array}{l}\text { Cuidados paliativos: o valor } \\
\text { da pessoa e sua história no } \\
\text { HUPE }\end{array}$ & $\begin{array}{l}\text { Hennemann-Krause, L. et } \\
\text { al }\end{array}$ & $\begin{array}{l}\text { Proporcionando a esses pacientes maiores possibilidades de } \\
\text { interação com seus familiares e de convivência social }\end{array}$ \\
\hline $\begin{array}{l}\text { Cuidados paliativos, } \\
\text { diagnósticos e terminalidade: } \\
\text { indicação e início do } \\
\text { processo de paliação. }\end{array}$ & $\begin{array}{l}\text { Rodrigues, LA ; Ligeiro, } \\
\text { C.; Silva, M. }\end{array}$ & $\begin{array}{l}\text { Engolir não é a mais importante para pacientes paliativos, mas } \\
\text { mudanças na deglutição podem colocar sua saúde em risco. }\end{array}$ \\
\hline $\begin{array}{l}\text { Contexto do } \text { cuidado } \\
\text { fisioterapêutico }\end{array}$ & Silva, LES, et. al. & $\begin{array}{l}\text { Ao receber cuidados dos profissionais, além de promover o } \\
\text { salvamento das relações interpessoais, o paciente também tende a } \\
\text { ter uma visão de melhora, prolongar sua vida com qualidade, } \\
\text { compreender sua real condição clínica, suas visões de futuro e } \\
\text { autonomia própria }\end{array}$ \\
\hline $\begin{array}{l}\text { A vivência da fonoaudiologia } \\
\text { na equipe de cuidados } \\
\text { paliativos de um Hospital } \\
\text { Universitário do Rio de } \\
\text { Janeiro }\end{array}$ & $\begin{array}{l}\text { Calheiros, } \\
\text { Albuquerque, CL. }\end{array}$ & $\begin{array}{l}\text { Podem permitir que a comunidade intelectual realize um } \\
\text { desenvolvimento epistemológico rigoroso e uma mudança, } \\
\text { garantindo assim a eficácia e a importância } \\
\text { social dos serviços prestados à sociedade }\end{array}$ \\
\hline $\begin{array}{l}\text { Ortotanásia em unidade de } \\
\text { terapia intensiva sob a ótica } \\
\text { dos profissionais }\end{array}$ & $\begin{array}{l}\text { Maia, FV.; Santos, TR.; } \\
\text { Ribeiro, I.M. }\end{array}$ & $\begin{array}{l}\text { Os idosos também mostram que o atendimento fonoaudiológico na } \\
\text { rede pública de saúde e os agravos no Brasil também estão se } \\
\text { desenvolvendo }\end{array}$ \\
\hline $\begin{array}{l}\text { Representação social da } \\
\text { Psicologia Hospitalar para } \\
\text { familiares de pacientes } \\
\text { hospitalizados em Unidade } \\
\text { de Terapia Intensiva }\end{array}$ & $\begin{array}{lr}\text { Moreira, } & \text { EKCB.; } \\
\text { Martins, } & \text { TM.; } \\
\text { MM }\end{array}$ & $\begin{array}{l}\text { A atuação do fonoaudiólogo tenha se ampliado, possibilitando } \\
\text { delinear ações e medidas preventivas adequadas para populações } \\
\text { específicas, desde que bem conhecidas }\end{array}$ \\
\hline
\end{tabular}

Fonte: Banco de dados Capes, Scholar e Scielo.

Neste quadro, destacaram-se os principais pontos abordados nos artigos, e após uma leitura criteriosa, conforme as similaridades semânticas do conteúdo criaram-se categorias, como se segue, entretanto os artigos que não se enquadraram nestas, foram analisados separadamente.

Conforme Ferreira, et.al (2014); Silva et. al. (2012); Alves (2010); Chen (2012) e Maia et. al. (2015) a primeira importância da atuação do profissional de fonoaudiologia na atuação em home care está diretamente relacionado com o público a ser atendido, sendo que quando o paciente é idoso é de grande valia que o fonoaudiólogo realizae o atendimento na casa do idoso.

A segunda importancia do atendimento do fonoaudiologo em home care, decorrre da ideia de Galassi (2014) ao defender a ideia de que a atenção domiciliar é uma alternativa ao cuidado hospitalar, possibilitando a retomada do lar como espaço para produção de cuidado, fundamentada nos princípios da humanização e da clínica ampliada, evitando hospitalizações desnecessárias e possibilitando um uso mais adequado dos recursos disponíveis. 
Uma terceira importância da atuação dos fonoaudiólogos em home care, conforme Cardoso (2012), consiste em pacientes que se encontram gravemente enfermos, tornando bastante complicada a ida desses pacientes até o hospital, sendo atendido no ambiente doméstico.

Já Hennemann-krause (2015) defende a ideia de que a atuação fonoaudiológica em home care contribui principalmente no aspecto psíquico, proporcionando a esses pacientes maiores possibilidades de interação com seus familiares e de convivência social.

Uma quarta importância da atuação do fonoaudiólogo consiste na ideia de Rodrigues et. al. (2015), quem defende que a atuação no processo de engolir não é a mais importante para pacientes paliativos, mas mudanças na deglutição podem colocar sua saúde em risco.

\section{Considerações Finais}

$\mathrm{O}$ atendimento domiciliar consiste numa gama de serviços realizados no domić́lio e destinados ao suporte terapêutico do paciente. Os principais objetivos deste tipo de atendimento são: contribuir para a otimização dos leitos hospitalares e do atendimento ambulatorial, visando à redução de custos; reintegrar o paciente em seu núcleo familiar e de apoio; proporcionar assistência humanizada e integral, por meio de uma maior aproximação da equipe de saúde com a família; estimular uma maior participação do paciente e de sua família no tratamento proposto; promover educação em saúde; ser um campo de ensino e pesquisa. Seus potenciais benefícios seriam a diminuição das re-internações e dos custos hospitalares, a redução do risco de infecção hospitalar e a manutenção do paciente no núcleo familiar e o aumento da qualidade de vida deste e de seus familiares.

Analisando todos os objetivos específicos propostos no início, pode-se concluir que sendo a visita domiciliar um atendimento realizado com objetivo de avaliar as necessidades do paciente, de seus familiares e do ambiente onde vivem, para estabelecer um plano assistencial voltado à recuperação e/ou reabilitação. São realizadas orientações às pessoas responsáveis pela continuidade do cuidado no domić́lio; o atendimento domiciliar, no qual ocorrem atividades assistenciais exercidas por profissionais e/ou equipe de saúde na residência do cliente, para executar procedimentos mais complexos, que exigem formação técnica para tal; e a internação domiciliar que são atividades assistenciais especializadas, exercidas por profissionais e/ou equipe de saúde na residência do cliente, com oferta de recursos humanos, equipamentos, materiais e medicamentos, assemelhando-se ao cuidado oferecido em ambiente hospitalar (instalação de um mini hospital).

Podendo analisar então que, como as diretrizes para atenção domiciliar são abrangentes e facilmente adaptáveis às necessidades de cada município ou região, a atuação do fonoaudiólogo ou do profissional de saúde pode variar em função do tipo de intervenção a ser realizada e da complexidade do caso a ser atendido. Sedo assim, as apresentações presenciais de fonoaudiólogos ou profissionais de saúde estão se tornando mais comuns no brasil.

\section{Referências}

Alves, D. B., \& Barbosa, M. T. S. (2010). Desigualdades na mortalidade por doenças crônicas entre idosos e sua associação com indicadores socioeconômicos no Brasil. Passo Fundo: Revista Brasileira de Ciências de Envelhecimento Humano.

Borges, M. V., et al. (2016). Implicações simbólicas na organização de um home care: interpretações entre a equipe de saúde e os cuidadores familiares. REAd. https://bit.ly/3r0ZhAb.

Braga, P. P., et. al. (2016). Oferta e demanda na atenção domiciliar em saúde (2016). Ciência \& Saúde Coletiva. https://bit.ly/2WeEs6q.

Brasil (2013). Portaria N 963, de 27 de maio de 2013, redefine a atenção domiciliar no âmbito do Sistema Único de Saúde (SUS). Brasília DF: Diário Oficial da República Federativa do Brasil, 2013. Disponível em: https://bit.ly/3nzKf2B.

Caheiros, A. S.; \& Albuquerque, C. L. (2012). A vivência da fonoaudiologia na equipe de cuidados paliativos de um Hospital Universitário do Rio de Janeiro. Rio de Janeiro: Revista Hospital Universitário Pedro Ernesto. 
Chen, B. C., et al (2012). Hemorrhagic complications associated with dabigatran use. Clinical toxicology.

Cardoso, M. C. A. F. (2012). Presbifagia: estado da arte da deglutição do idoso. Passo Fundo: Revista Brasileira de Ciências do Envelhecimento Humano, 2012. Disponível em: https://bit.ly/3acAoLY.

Fereira, F. P. C.; Bansi, L. O.; \& Paschoal, S. M. P. (2014). Aged care services and home health care and institutional strategies. Revista Brasileira de Geriatria e Gerontologia.

Fontoura, V. T.; \& Arend, S. C. (2015). Redes organizacionais como alternativa para gestão hospitalar: o sindicato dos hospitais beneficentes, religiosos e filantrópicos do Vale do Rio Pardo (SINDHVARP). Revista Brasileira de Desenvolvimento Regional.

Galassi, C. V., et. al. (2014). Atenção domiciliar na atenção primária à saúde: uma síntese operacional. Santo André: ABCS Health Sciences, 2014. https://bit.ly/3gRqYab.

Genet, N., et. al. (2011). Home care in Europe: a systematic literature review. Woolloongabba: MC Health Services Research, 2011. https://bit.ly/3msPAr6.

Goulart, B. N. G. (2010). Fonoaudiologia e promoção da saúde: relato de experiência baseado em visitas domiciliares. Campinas: CEFAC, 2010. https://bit.ly/3qYVgwm.

Hennemann-Krause, L., et. al. (2015). Cuidados paliativos: o valor da pessoa e sua história no HUPE. Revista Hospital Universitário Pedro Ernesto, 2015.

Maia, F. V.; Santos, T. R.; \& Ribeiro, I. M. (2015). Ortotanásia em unidade de terapia intensiva sob a ótica dos profissionais. Catanduva: CuidArte Enfermagem.

Malta, D. C.; \& Merhy, E. E. (2010). O percurso da linha do cuidado sob a perspectiva das doenças crônicas não transmissíveis. Interface.

Mansur, L. L.; \& Mchado, T. H. (2010). Afasias: uma visão multidimensional da atuação do fonoaudiólogo. ROCA.

Moreira, E. K. C. B.; Martins, T. M.; \& Castro, M. M. (2012). Representação social da Psicologia Hospitalar para familiares de pacientes hospitalizados em Unidade de Terapia Intensiva. Revista Sociedade Brasileira de Psicologia Hospitalar.

Moreira, T. N. F., et. al. (2014). The foundation of care: family health program teams dealing with domestic violence situations. Saúde \& Sociedade.

Padovani, A. R. (2013). Avaliação clínica da deglutição em unidade de terapia intensiva. CoDAS.

Pereira, F. C. B., et. al. (2011). The history of speech-language pathology in Minas Gerais state: the main characters' impression. CEFAC.

Rodriges, L. A.; Ligeiro, C.; \& Silva, M. (2015). Cuidados paliativos, diagnósticos e terminalidade: indicação e início do processo de paliação. Catan duva: CuidArte Enfermagem.

Savassi, L. C. M. (2011). Iniciação à prática de ESF. Faculdade Senac.

Ribeiro, R. A. B. (2011). Internação Domiciliar no SUS: breve histórico e desafios sobre sua implantação no Distrito Federal. Brasília: Revista Comunicação em Ciências da Saúde.

Silva, K. L., et. al. (2017). Por que é melhor em casa? A percepção de usuários e cuidadores da atenção domiciliar. Cogitare Enfermagem.

Silva, L. E. S., et al. (2013). Contexto do cuidado fisioterapêutico: reveses e vieses na inserção comunitária à atenção domiciliar. Revista Kairós Gerontologia.

Silveira, M. H.; Ciampone, M. H. T., \& Gutierrez, B. A. O. (2014). Percepção da equipe multiprofissional sobre cuidados paliativos. Revista Brasileira de Geriatria e Gerontologia.

Stewart, M., et. al. (2010). Medicina centrada na pessoa: transformando o método clínico. Artmed..

Vasconcelos, J. F., et al. (2015). Desospitalização para cuidado domiciliar: impactos clínico e econômico da linezolida. Jornal Brasileiro de Economia da Saúde.

Wachs, L. S., et al. (2016). Prevalence of home care and associated factors in the Brazilian elderly population. Cadernos de Saúde Pública.

Zeppe, M. A. (2014). A inserção do psicólogo no sistema único de saúde (SUS): do usuário ao sujeito. Ijuí: Universidade Regional do Noroeste do Estado do Rio Grande do Sul. 